\title{
VELOCITY MODEL BUILDING USING PRE-STACK DEPTH MIGRATION
}

C. KOENINGER, N. JONES and S. DEREGOWSKI Western Geophysical, 455 London Road, Isleworth, Middlesex TW7 5AB, UK

Time migration algorithms prove to be inadequate when trying to correctly image seismic sections in areas of complex geology. In such media, velocities vary both vertically and laterally giving rise to non-hyperbolicity in the observed moveout of the seismic reflections. To deal with this situation, a depth migration must be employed. However, a dilemma arises in that the performance of the pre-stack depth migration is sensitive to errors in the velocity model supplied by the user, that is the more accurate the underlying velocity model, the better the migrated image. This sensitivity provides an excellent tool for the iterative building of depth models (Figures $1 \& 2$ ). We present a method that combines pre-stack depth migration with velocity analysis and mapmigration.

Velocity Model Building

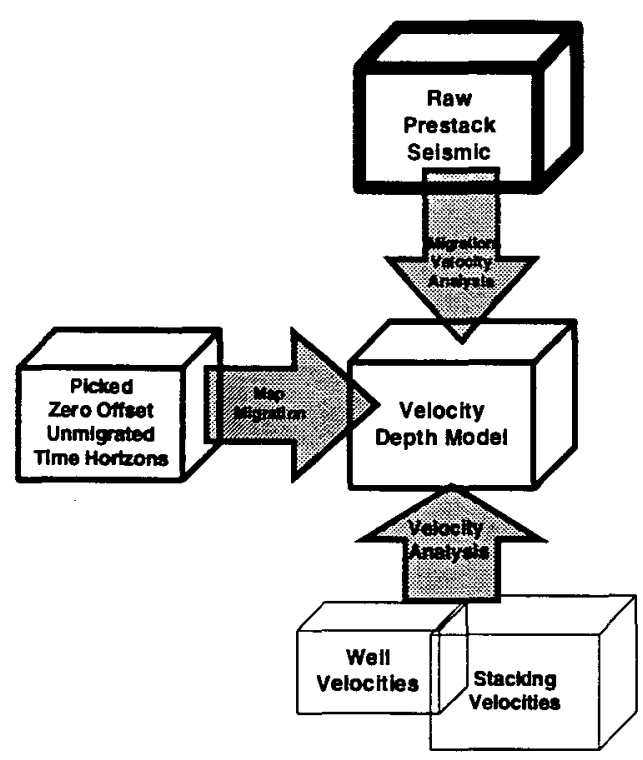

Figure 1

\section{Imaging Strategy}



Figure 2

With the possible exception of model verification the proposed method works in an iterative, top-down fashion. Initially zero-offset (normal incidence) time horizons are map-migrated using a 'best-guess' interval velocity model. For the first iteration, this function would vary only in the near surface, while retaining a constant value for the 
rest of the model. Given any surface location below which velocity analysis is required over a specified depth window a fan of rays is traced upwards through the model from 'diffractors' spaced at regular depth intervals. This results in a table of ray attributes (travel-times, emergence angles, radii of wavefront curvature) described as functions of in-line and cross-line take-off angles (Figure 3). One advantage of using cross-line and in-line angles is that 2D geometries can be handled as a special case of 3D.

\section{Ray Co-ordinates}

\section{'In-line' angle $\alpha$ \\ 'Cross-line' angle $\phi$}

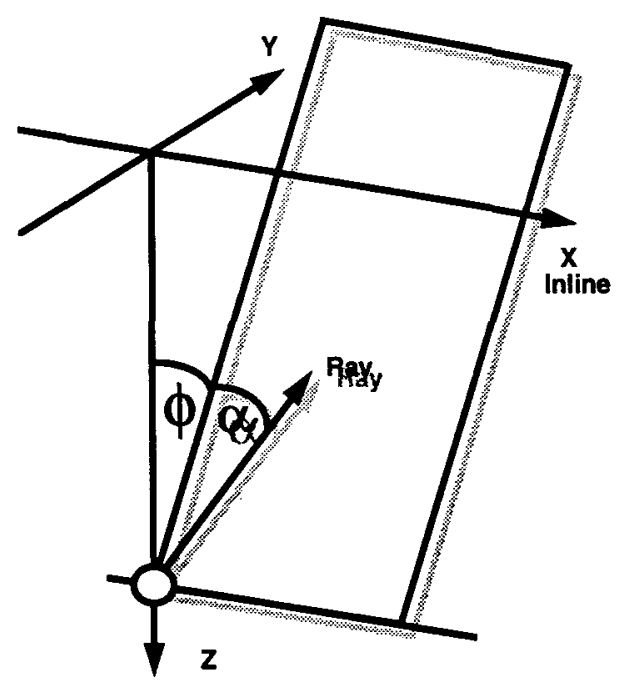

Partial Aperture Images

Input CMP Bins

Output CDP Location

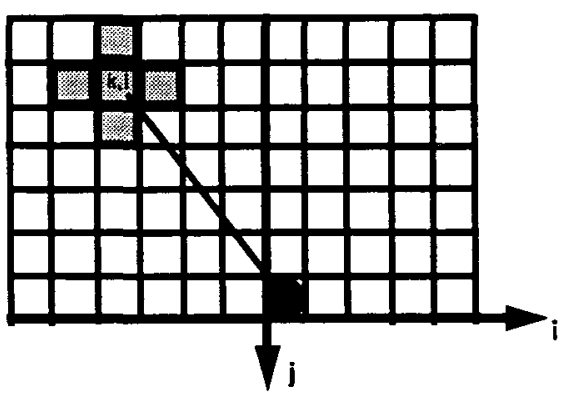

$R_{1}(x, z):$ ij'th partial aperture image; using a restricted range of input CMPs

$R(x, z)=\Sigma_{1 j} R_{1 j}(x, z) \quad$ Full aperture image

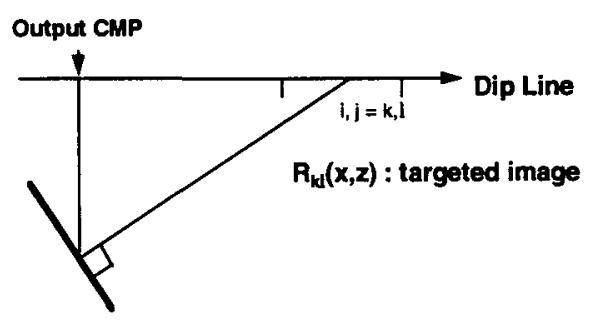

Figure 4

Figure 3

This raytrace table is then used to drive a common-offset, Kirchhoff-type depth migration in order to produce migrated unstacked common reflector point (CRP) gathers from which the model implied moveout corrections have been removed. The traces within each CRP gather are presented in terms of vertical travel time so that standard velocity analysis can be performed (Figure 5). However the picked velocities merely provide us with $\mathrm{rms}$ velocities down to each boundary at sparse surface locations. These have to converted to interval velocities, using assumed vertical gradients. This is very much an interpretational step and additional velocity information, such as well log data, is invaluable for the identification of such vertical velocity gradients. Well information also provides us to compute an 'anisotropy' correction factor to be used for the final vertical travel time to depth conversion. 
Having defined the new velocity function. the next iteration is initiated by mapmigrating the zero-offset time horizons again (Figure 2). This ensures the consistency of any subsequent, 'updated' model with the invariant normal incidence times. The previous steps are repeated until the map-migrated image converges on the model estimate at target depths, implying that the velocity structure of the overburden has been reasonably constrained.

The CRP gathers are themselves obtained by the explicit mixing of partial images as implied by the in-line and cross-line apertures (Figure 4). Such partial aperture images (Figure 6) not only allow for the imaging of weak steeply dipping events by appropriate 'beam steering' but also allow the computation to be distributed over several computational nodes. The technique also allows the predicted result of the prestack depth migration to be modelled and compared with the actual result (Figure 7). This allows us to judge how far down the local overburden has been correctly modelled. For accurate AVO work the raw images can also be divided by an equalising 'Fold' function which is shown in the right most portion of Figure 7. The Fold is computed from the acquisition geometry and assumed depth model.

The CRP gather can also be stacked to give a migrated trace which may be inserted into a post-stack depth migrated section or a pre-stack depth migrated section based on the Eikonal equation, in order to clarify the final image. An important aspect of this procedure is that only subsets of the original data volume need be migrated. The resultant savings in run-time thereby endorse the feasibility of this iterative, model building technique.

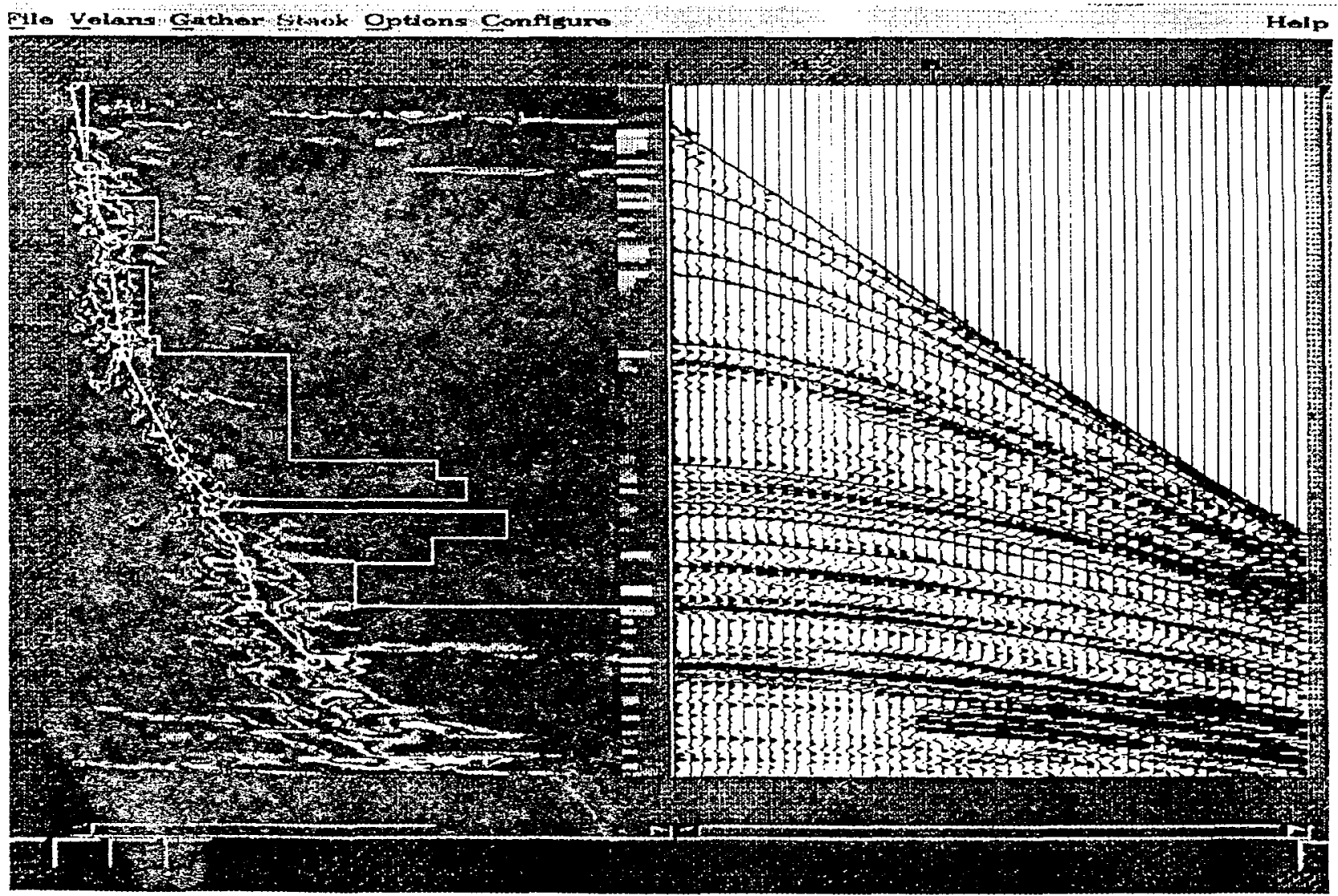

Fig. 5 - Velocity Analysis applied to depth migrated CRP gather 


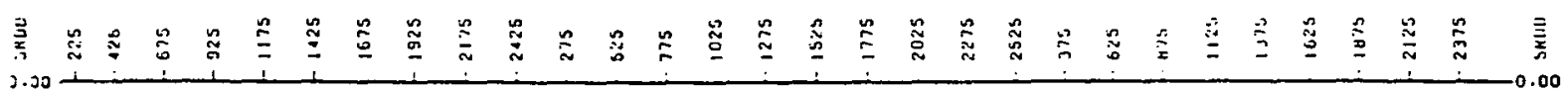

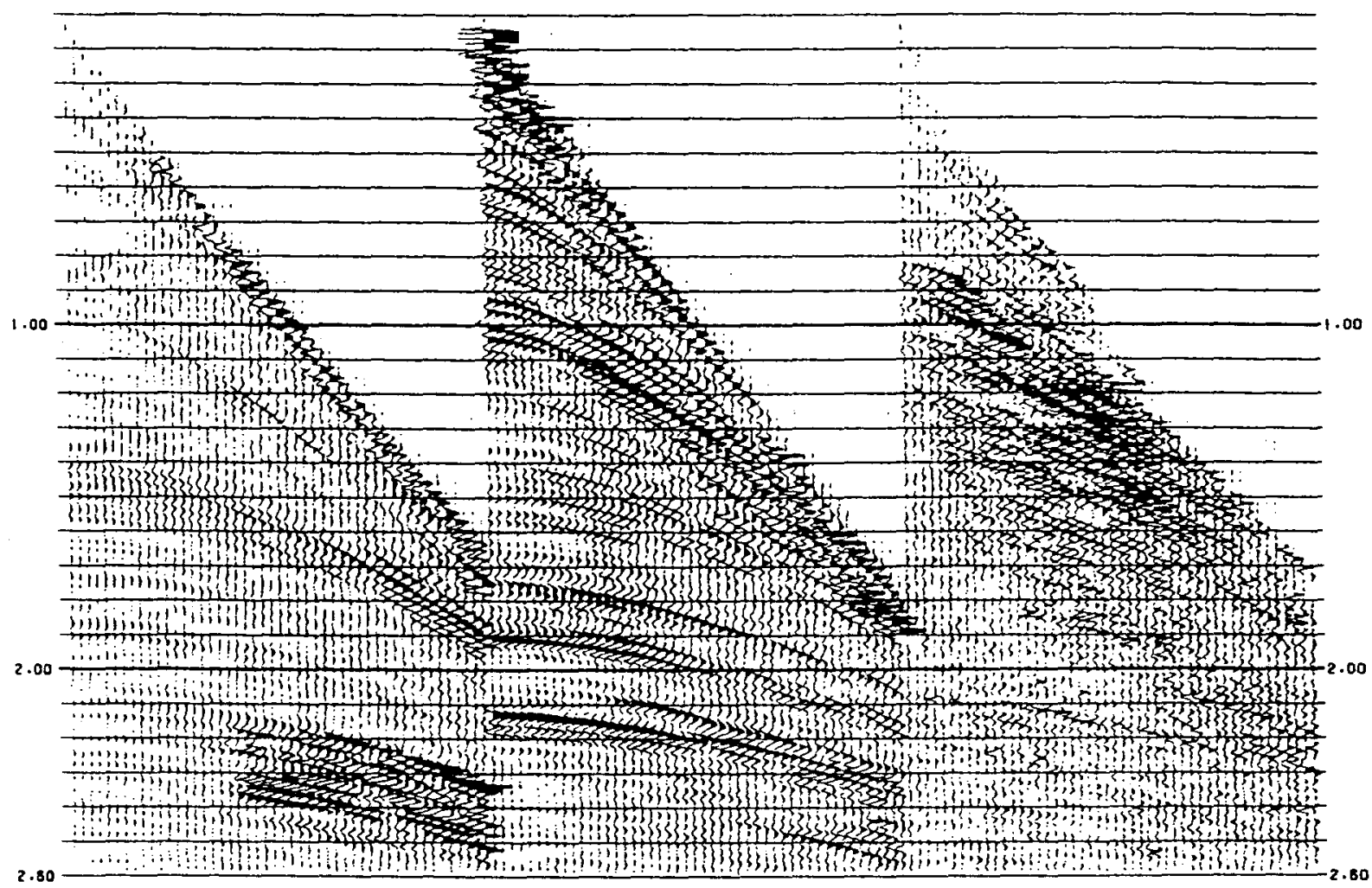

Fig. 6 - Partial Aperture Images (left and right images are for steep dips)

总

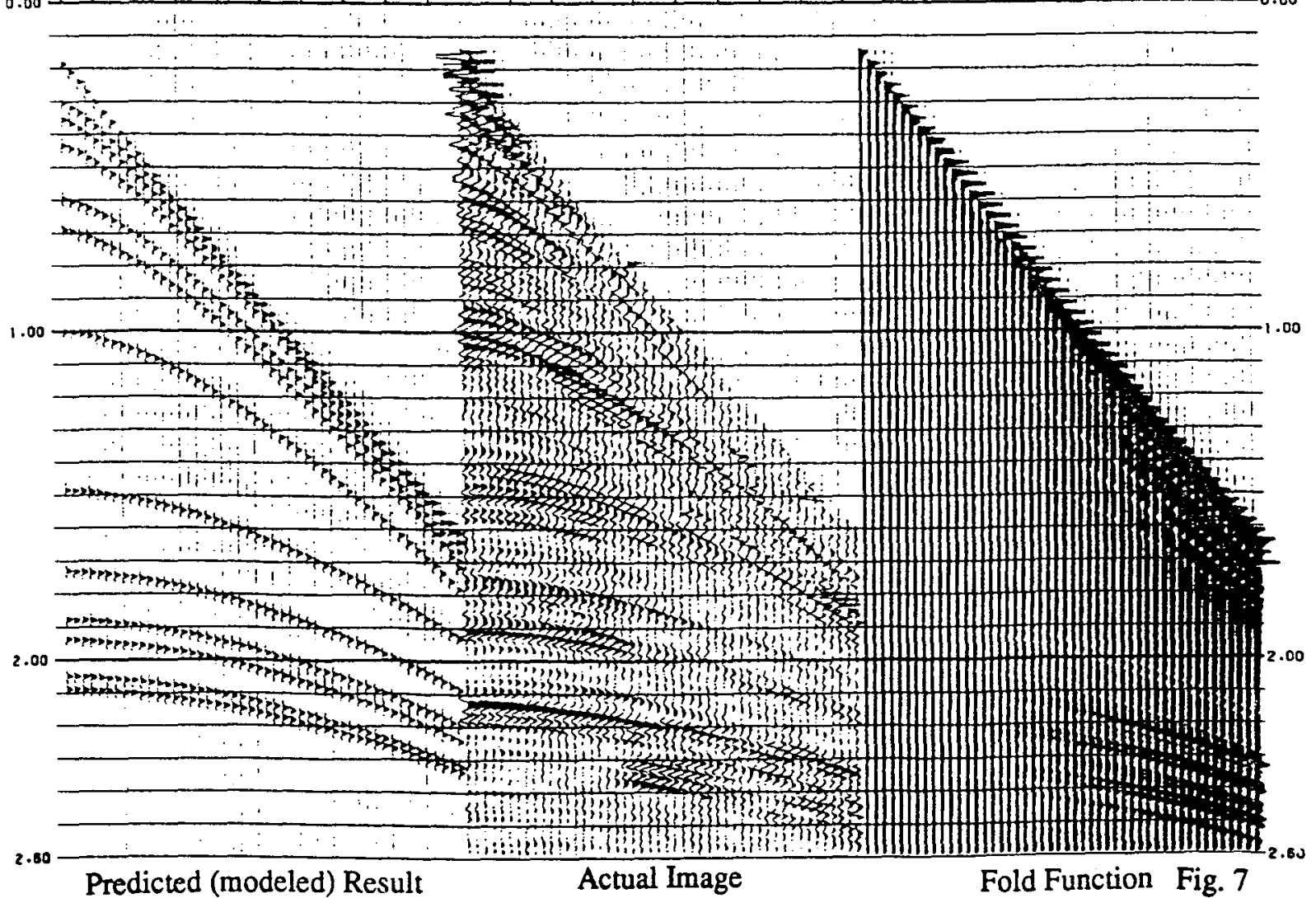

Predicted (modeled) Result

Actual Image

Fold Function Fig. 7 